\title{
ARTICLE Population-based volume kinetics of Ringer's lactate solution in patients undergoing open gastrectomy
}

Joo-Hyun Lee ${ }^{1}$, Yeong-Jin Choo ${ }^{2}$, Yong-Hun Lee ${ }^{3}$, Jin-Ho Rhim ${ }^{4}$, Soo-Han Lee ${ }^{5}$, Byung-Moon Choi ${ }^{3}$, Sung-Tae Oh ${ }^{6}$, Kyu-Taek Choi ${ }^{3}$ and Gyu-Jeong $\mathrm{Noh}^{3,7}$

In order to maintain stable blood pressure and heart rate during surgery, anesthesiologists need to administer the appropriate amount of fluid with appropriate fluid type to the patient, then quantifying how fluid is distributed and eliminated from the body is useful for establishing a fluid administration strategy. In this study we characterized the volume kinetics of Ringer's lactate solution in patients undergoing open gastrectomy. When propofol and remifentanil reached a pseudosteady state at the target concentration and blood pressure was stabilized following surgical stimulation, enrolled patients were administered $1000 \mathrm{~mL}$ of Ringer's lactate solution for $20 \mathrm{~min}$, followed by continuous infusion at a rate of $6 \mathrm{~mL} / \mathrm{kg} / \mathrm{h}$ until the time of the last blood collection for volume kinetic analysis. Arterial blood samples were collected to measure the hemoglobin concentration at different time points. The change in hemoglobin-derived plasma dilution induced by the administration of Ringer's lactate solution was evaluated by nonlinear mixed effects modeling. Three hundred and twenty-three plasma dilution data points from 27 patients were used to determine the pharmacokinetic characteristics of Ringer's lactate solution. A two-volume model best described the pharmacokinetics of Ringer's lactate solution. The mean arterial pressure (MAP) and body weight (WT) were significant covariates for the elimination clearance $\left(k_{r}\right)$ and central volume of distribution at baseline $\left(V_{c 0}\right)$, respectively. The parameter estimates were as follows: $k_{r}(\mathrm{~mL} / \mathrm{min})=124+(\mathrm{MAP} / 70)^{14.2}, V_{c 0}(\mathrm{~mL})=0.95+3440 \times(\mathrm{WT} / 63), V_{t 0}(\mathrm{~mL})=2730$, and $k_{t}(\mathrm{~mL} / \mathrm{min})=181 . \mathrm{A} \mathrm{higher}$ MAP was associated with a greater elimination clearance and, consequently, less water accumulation in the interstitium. As body weight increases, volume expansion in the blood vessels increases.

Keywords: anesthesiologist; crystalloid solution; fluid shifts; pharmacokinetics; mean arterial pressure; body weight

Acta Pharmacologica Sinica (2019) 40:710-716; https://doi.org/10.1038/s41401-018-0179-3

\section{INTRODUCTION}

It is important to maintain a stable blood pressure and heart rate during surgery. Anesthesiologists select the appropriate fluid type for the patient and administer the appropriate amount of fluid. Previous studies have shown that the type of fluid therapy is associated with postoperative recovery and prognosis [1-4]. For successful fluid therapy, it is helpful to quantify how the administered fluid is distributed and eliminated from the body. Volume kinetics, a method used to analyze the disposition of infusion fluids, differs from general drug pharmacokinetic models in terms of input data [5]. It is difficult to measure the plasma concentration using standard methods because water is a major component of the fluids and plasma [6]. However, the water content of the whole blood reflects the dilution of hemoglobin, which is easily measurable [5]. Thus, the dilution of hemoglobin may be used as an indirect indicator of the infusion fluid concentration $[5,6]$.
Because surgical patients show different physical characteristics, it is necessary to consider inter-individual variability when administering fluids. This inter-individual variability may be explained by population analysis [7]. The inclusion of covariates, such as body weight and blood pressure, in the parameters of the volume kinetic model may enable the individualized administration of fluids to patients. Previous studies have attempted to explain inter-individual variability using population analysis; however, the methods are not universal $[8,9]$.

The aim of this study was to characterize the volume kinetics of Ringer's lactate solution by nonlinear mixed effects modeling in patients undergoing open gastrectomy.

\section{MATERIALS AND METHODS}

Investigation fluid

Ringer's lactate solution (JW Pharmaceutical Co., Ltd, Seoul, Korea) was used in this study.

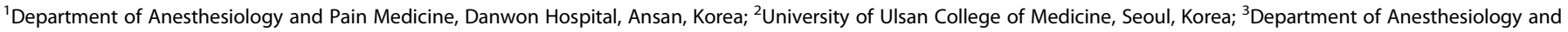

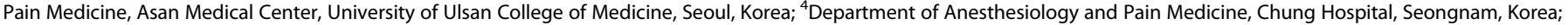

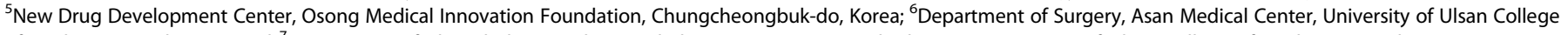
of Medicine, Seoul, Korea and ${ }^{7}$ Department of Clinical Pharmacology and Therapeutics, Asan Medical Center, University of Ulsan College of Medicine, Seoul, Korea Correspondence: Soo-Han Lee (vetingod@gmail.com) or Byung-Moon Choi (byungmoonchoi7@gmail.com)

These authors contributed equally: Joo-Hyun Lee, Young-Jin Choo
}

Received: 30 June 2018 Accepted: 26 September 2018

Published online: 16 October 2018 
Patient population

This clinical trial was approved by the Institutional Review Board of Asan Medical Center (2017-0177) and was registered on an international clinical trials registry platform (http://cris.nih.go.kr; KCT0002463). Written informed consent was obtained from all patients. Thirty-two patients undergoing open gastrectomy under general anesthesia were enrolled in this study. Patients were excluded if they had a history of hepatic, cardiopulmonary, or renal disease, were pregnant, had an estimated glomerular filtration rate of $<60 \mathrm{~mL} / \mathrm{min}$, or had preoperative hemoglobin levels $<9 \mathrm{mg} / \mathrm{dL}$. If the estimated blood loss exceeded $450 \mathrm{~mL}$ during the operation, the patient was withdrawn from the trial.

\section{Study procedure}

All patients fasted for 6-8 h prior to surgery. For continuous blood pressure monitoring and frequent sampling, a 20-gauge catheter was inserted into a radial artery. Electrocardiography, pulse oximetry, and monitoring of the partial pressure of end-tidal carbon dioxide $\left(\mathrm{P}_{\mathrm{ET}} \mathrm{CO}_{2}\right)$ and invasive blood pressure (DatexOhmeda S/5; Planar Systems, Inc., Beaverton, OR, USA) were continuously performed. The cardiac output and stroke volume variation (SVV) were assessed according to the algorithm within the Vigileo monitor (FloTrac/Vigileo ${ }^{\mathrm{TM}}$; Edwards Lifesciences, Irvine, CA, USA). Throughout surgery, these data were downloaded to personal computers using RS232C cables. The target concentrations of propofol and remifentanil were adjusted to maintain bispectral index (BIS ${ }^{\oplus}$; Covidien, Boulder, CO, USA) values of $<60$ and stable hemodynamic values (systolic blood pressure $>80$ $\mathrm{mmHg}$; heart rate $>45$ beats $/ \mathrm{min}$ ), respectively. The target concentration ranges of propofol and remifentanil were 2.5-3 $\mu \mathrm{g} / \mathrm{mL}$ and $3-20 \mathrm{ng} / \mathrm{mL}$, respectively. If necessary, ephedrine or atropine was administered to maintain stable hemodynamic values. Tracheal intubation was performed following the administration of rocuronium $(0.6 \mathrm{mg} / \mathrm{kg})$. The lungs of the patients were then ventilated with oxygen in air $(1: 2$, fraction of inspired oxygen $\left.\left[\mathrm{FiO}_{2}\right]=0.47\right)$, and the tidal volume and ventilation rate were adjusted to maintain the $\mathrm{P}_{\mathrm{ET}} \mathrm{CO}_{2}$ between 35 and $45 \mathrm{mmHg}$. Neuromuscular blockade was reversed by administering neostigmine and glycopyrrolate at the end of surgery.

Administration of Ringer's lactate solution

When propofol and remifentanil reached a pseudosteady state at the target concentration and the blood pressure was stabilized following surgical stimulation, Ringer's lactate solution infusion for volume kinetic analysis was initiated. The patients were administered $1000 \mathrm{~mL}$ of Ringer's lactate solution for $20 \mathrm{~min}$, followed by continuous infusion at a rate of $6 \mathrm{~mL} / \mathrm{kg} / \mathrm{h}$ until the time of the last blood collection for volume kinetic analysis. When the SVV exceeded $13 \%$, the SVV was maintained below $13 \%$ by increasing the infusion rate of Ringer's lactate solution. All infusions of Ringer's lactate solution were performed using infusion pumps (Baxter Flo-Gard 6201, Deerfield, IL, USA). During the clinical trial for volume kinetic analysis, no other fluids were administered with the exception of Ringer's lactate solution.

\section{Measurement of hemoglobin, estimated blood loss, and urine output}

Arterial blood samples $(1 \mathrm{~mL})$ were collected to measure the hemoglobin concentration and hematocrit at preset intervals: 0, 5, $10,15,20,25,30,40,60,90,120$, and 180 min after initiating the administration of $1000 \mathrm{~mL}$ of Ringer's lactate solution. Blood tests were conducted at the clinical laboratories of Asan Medical Center, which is certified by the College of American Pathologists and Korean Society for Laboratory Medicine. The intraoperative estimated blood loss was calculated as the sum of the volumes of blood contained in suction systems and gauzes [10]. Urine output was also monitored with a Foley catheter placed in the bladder during the sampling period.
Hemoglobin-derived plasma dilution

The hemoglobin-derived plasma dilution is used to indicate the dilution of plasma in the central body fluid space expanded by the infused fluid $\left(v_{c}(t)-V_{c}\right) / V_{c}$ [5]. The reference equation for this relationship may be expressed as follows [5]:

$\frac{v_{c}(t)-V_{c}}{V_{c}}=\frac{\frac{\mathrm{Hb}}{\mathrm{Hb}(t)}-1}{1-\mathrm{Hct}}$,

where $v_{c}$ is the size of the expanded central body plasma fluid space, $V_{c}$ is the baseline plasma fluid space, Hct is the hematocrit, and $\mathrm{Hb}$ is the hemoglobin concentration in the whole blood at baseline or time $(t)$ [5].

Population pharmacokinetic analysis

Population pharmacokinetic analysis was performed with NONMEM VII level 4 (ICON Development Solutions, Ellicott City, MD, USA). Plasma dilutions were fitted to one- or two-volume models using the ADVAN13 subroutines and first-order conditional estimation with interaction. The ADVAN13 is useful for stiff and nonstiff equations. Moreover, the ADVAN13 differential equation solver has been shown to solve problems more rapidly with the new estimation methods, whereas ADVAN6 or 9 may continue to have a greater advantage for classical NONMEM methods [11].

The differential equation of the one-volume model is as follows [6]:

$$
\frac{\mathrm{d} v(t)}{\mathrm{d} t}=k_{i}-k_{b}-k_{r} \times\left(\frac{v(t)-V_{0}}{V_{0}}\right),
$$

where $k_{i}$ is the infusion rate of fluid, $k_{b}$ is the baseline fluid loss, including insensible loss, $k_{r}$ is the elimination clearance, $v$ is the expanded body fluid space at the central compartment, and $V_{0}$ is the baseline fluid space at the central compartment.

The differential equation of the two-volume model is as follows [6]:

$$
\begin{gathered}
\frac{\mathrm{d} v_{c}(t)}{\mathrm{d} t}=k_{i}-k_{b}-k_{r} \times\left(\frac{v_{c}(t)-V_{c 0}}{V_{c 0}}\right)-k_{t} \times\left(\frac{v_{c}(t)-V_{c 0}}{V_{c 0}}\right)+k_{t} \times\left(\frac{v_{t}(t)-V_{t 0}}{V_{t 0}}\right), \\
\frac{\mathrm{d} v_{t}(t)}{\mathrm{d} t}=k_{t} \times\left(\frac{v_{c}(t)-V_{c 0}}{V_{c 0}}\right)-k_{t} \times\left(\frac{v_{t}(t)-V_{t 0}}{V_{t 0}}\right)
\end{gathered},
$$

where $k_{t}$ is the distributional clearance, $v_{c}$ and $v_{t}$ are expanded body fluid spaces at the central and peripheral compartments, respectively, and $V_{c 0}$ and $V_{t 0}$ are baseline fluid spaces at the central and peripheral compartments, respectively. $k_{b}$ was set to $0.8 \mathrm{~mL} / \mathrm{min}$ without estimating [12].

The inter-individual random variabilities of the pharmacokinetic parameters were estimated assuming a log-normal distribution. Diagonal matrices were estimated for the various distributions of $\eta$, where $\eta$ represents the inter-individual random variability with a mean of zero and variance of $\omega^{2}$. Additive and constant coefficients of variation and combined additive and constant coefficient of variation residual error models were evaluated during the model building process. NONMEM computed the minimum objective function value (OFV), a statistical equivalent to the $-2 \log$ likelihood of the model. An $a$-level of 0.05 , which corresponds to a reduction in the OFV of 3.84 (chi-square distribution, degree of freedom $=1, P<0.05)$, was used to distinguish between hierarchical models [11]. The covariates analyzed included age, sex ( $0=$ female, $1=$ male), body weight, height, body surface area [13], lean body mass [14], ideal body weight [15], body mass index, cardiac output, cardiac index, stroke volume, SVV, stroke volume index, heart rate, systolic arterial pressure, diastolic arterial pressure, and mean arterial pressure. A non-parametric bootstrap analysis served to internally validate the models (fit4NM 3.3.3, Eun-Kyung Lee and Gyu-Jeong Noh; http:// cran.r-project.org/web/packages/fit4NM/index.html; last accessed 
16 March 2011) [16]. Predictive checks were also performed using fit4NM 3.3.3 [7]. The levels of significance of the covariates were further assessed using a randomization test that randomly permutes the covariate in the original dataset using fit4NM 3.3.3. The differences in the OFVs between the reference (without the covariate tested) and covariate (including the covariate tested) models fitted to the permuted datasets were sorted in ascending order. The value corresponding to the 5 th percentile was set as the $\delta$-value. If a change in the OFVs between the reference and covariate models fitted to an original dataset was greater than the $\delta$-value, it was considered to be good evidence that the covariate effect was statistically significant $[16,17]$. Deterministic simulations were performed to characterize the effects of the covariates on volume expansion in the central and peripheral fluid spaces using the estimated pharmacokinetic parameters of the final model.

Statistical analysis

Statistical analyses were conducted using $\mathrm{R}$ (version 3.4.4; R Foundation for Statistical Computing, Vienna, Austria) or SigmaStat version 3.5 for Windows (Systat Software, Inc., San Jose, CA, USA). Data are expressed as the mean \pm standard deviation for normally distributed continuous variables, median (25\%-75\%) for nonnormally distributed continuous variables, and counts and percentages for categorical variables.

\section{RESULTS}

Of the 32 patients enrolled, 5 patients were excluded from the analysis because of the withdrawal of consent $(n=1)$, early termination of surgery because of peritoneal seeding $(n=1)$, and bleeding greater than $450 \mathrm{~mL}$ during the operation $(n=3)$. The characteristics of the remaining 27 patients are summarized in Table 1. A plasma dilution at $20 \mathrm{~min}$ after the time of initiating the administration of $1000 \mathrm{~mL}$ of Ringer's lactate solution was 0.50 in one patient. This value was more than twice the maximal plasma dilution of the other patients. The plasma dilution values measured before $(15 \mathrm{~min})$ and after $(25 \mathrm{~min})$ this time were 0.19 and 0.17 , respectively. Moreover, no excessive fluid was administered at this time. This was therefore considered to be a measurement error and was excluded from the population pharmacokinetic analysis. In total, 323 hemoglobin-derived plasma dilution data points from 27 patients were used to determine the pharmacokinetic characteristics of Ringer's lactate solution.

The time courses of plasma dilution during and after the intravenous infusion of $1000 \mathrm{~mL}$ of Ringer's lactate solution over $20 \mathrm{~min}$ are shown in Fig. 1a. The dilution increased until $20 \mathrm{~min}$ after initiating the administration of $1000 \mathrm{~mL}$ of Ringer's lactate solution and then decreased. Twelve patients had negative plasma dilution values during the sampling period. A two- volume model best described the pharmacokinetics of Ringer's lactate solution in surgical patients undergoing open gastrectomy. Parameter estimates of the competing base and covariate pharmacokinetic models of Ringer's lactate solution are described in Table 2. Changes in the mean arterial pressure (MAP) and heart rate over time during surgery are presented in Fig. 2. The MAP tended to increase during the administration of $1000 \mathrm{~mL}$ of Ringer's lactate solution, and after the rapid administration, the MAP tended to gradually decrease until the end of the operation. The MAP was a significant covariate for the elimination clearance $\left(k_{r}\right)$ (Eq. (4)) and resulted in an improvement in the OFV $(20.13, P<$ 0.01 , degree of freedom $(d f)=1)$, compared to the basic model (number of model parameters $=8$ ).

$k_{r}=124+(\operatorname{MAP} / 70)^{14.2}$.

Body weight (WT) was also a significant covariate for the central volume of distribution at baseline $\left(V_{c 0}\right)($ Eq. (5)) and led to a further improvement in the $\operatorname{OFV}(6.23, P=0.013, \mathrm{df}=1)$ compared to the OFV of a pharmacokinetic model that included the $\mathrm{Cl}$ as a covariate for the $k_{r}$ only (number of model parameters $=9$ ). The $\delta$ value between the covariate (MAP on $k_{r}$ ) and final pharmacokinetic (MAP on $k_{r}$ and WT on $V c_{0}$ ) models was 2.49.

$V_{c 0}=0.95+3440 \times(\mathrm{WT} / 63)$.

\begin{tabular}{|ll}
\hline \multicolumn{2}{l}{ Table 1. Characteristics of the study patients $(n=27)$} \\
\hline Male/female & $15 / 12$ \\
Age (year) & $57.0 \pm 11.4(36-87)$ \\
Weight $(\mathrm{kg})$ & $45.4 \pm 6.9(40.0-78.0)$ \\
Height $(\mathrm{cm})$ & $161.9 \pm 8.4(146.4-161.5)$ \\
$\mathrm{BMI}\left(\mathrm{kg} / \mathrm{m}^{2}\right)$ & $24.0 \pm 2.7(18.3-28.7)$ \\
$\mathrm{BSA}\left(\mathrm{m}^{2}\right)$ & $1.7 \pm 0.2(1.3-1.9)$ \\
LBM $(\mathrm{kg})$ & $45.4 \pm 9.7(27.4-57.7)$ \\
IBW $(\mathrm{kg})$ & $57.7 \pm 7.6(45.0-69.7)$ \\
ASA PS $1 / 2$ & $7 / 20$ \\
Duration of anesthesia & $178.6 \pm 19.8(145-210)$ \\
Baseline hemoglobin $(\mathrm{mg} / \mathrm{dL})$ & $11.9 \pm 1.3(9.5-14.3)$ \\
Baseline hematocrit $(\%)$ & $35.7 \pm 3.7(29.7-42.6)$ \\
\hline
\end{tabular}

Data are presented as counts or means \pm SDs (with ranges) as appropriate. ASA PS American Society of Anesthesiologists physical status, $B M I$ body mass index, $B S A$ body surface area calculated using the variation of DuBois and DuBois [13], LBM lean body mass calculated using the Janmahasatian formula [14], IBW ideal body weight calculated using the Robinson formula [15]
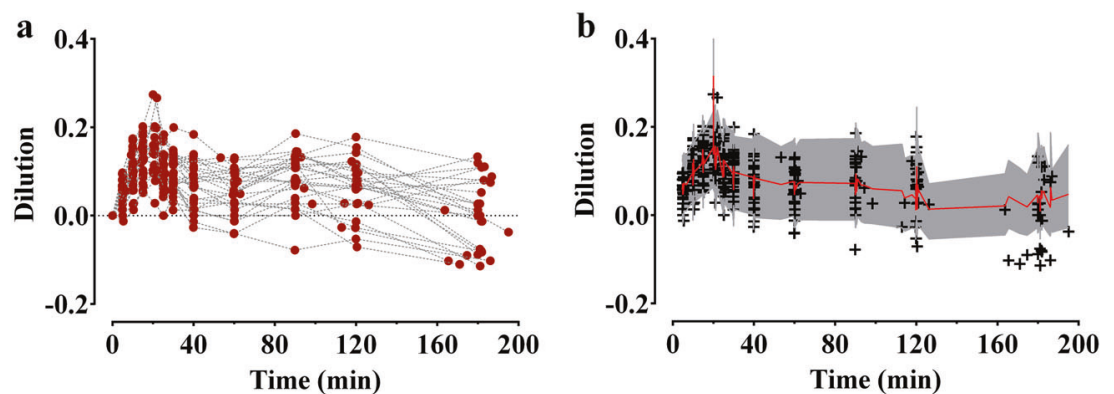

Fig. 1 Plasma dilution (a) during and after intravenous infusion of $1000 \mathrm{~mL}$ of Ringer's lactate solution over 20 min in 27 patients undergoing open gastrectomy and predictive checks (b) of the final pharmacokinetic model for Ringer's lactate solution. The red solid line and shaded areas indicate the $50 \%$ prediction line and $90 \%$ prediction intervals, respectively. + : observed dilution. The time point at which $1000 \mathrm{~mL}$ of Ringer's lactate solution was initiated was set as the reference time 0 
Table 2. Parameter estimates (RSE, \%CV) of competing basic and covariate pharmacokinetic models of Ringer's lactate solution

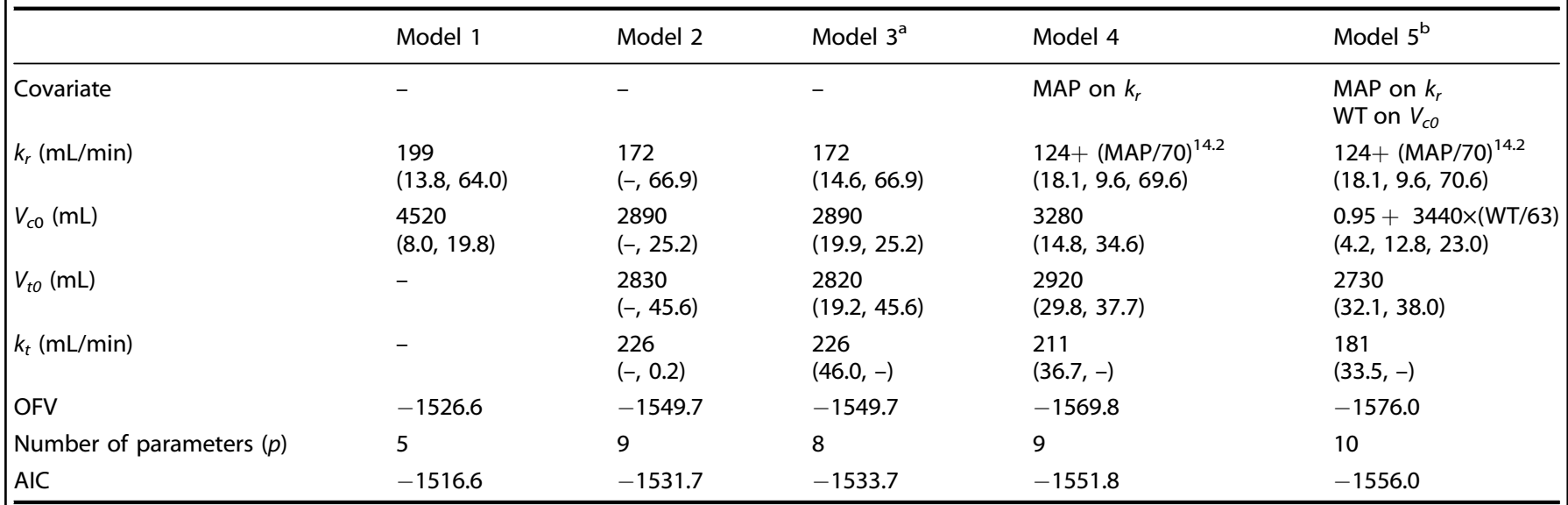

OFV objective function value ( $-2 \mathrm{log}$ likelihood, $-2 \mathrm{LL})$, AIC Akaike information criteria $(-2 \mathrm{LL}+2 \times p), C V$ coefficient of variation, $R S E$ relative standard error $=$ $\mathrm{SE} /$ estimate $\times 100(\%)$, MAP mean arterial pressure, $W T$ actual body weight, $k_{r}$ elimination clearance $(\mathrm{mL} / \mathrm{min}), V_{c 0}$ central volume of distribution at baseline $(\mathrm{mL}), V_{t o}$ peripheral volume of distribution at baseline $(\mathrm{mL}), k_{t}$ distributonal clearance between central and peripheral compartment $(\mathrm{mL} / \mathrm{min})$

aselected basic model

belected final model
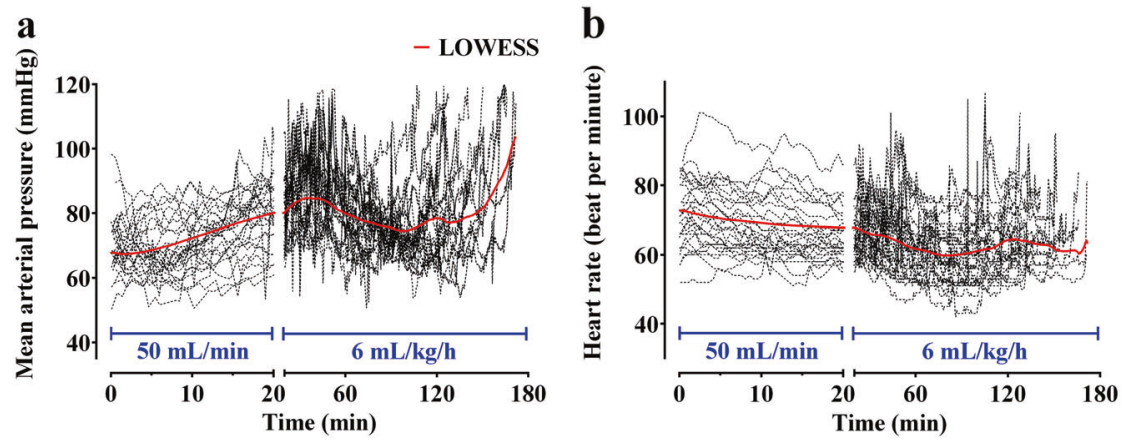

Fig. 2 Changes in mean arterial pressure (a) and heart rate (b) over time during surgery. Patients were administered $1000 \mathrm{~mL}$ of Ringer's lactate solution for $20 \mathrm{~min}$ (infusion rate of $50 \mathrm{~mL} / \mathrm{min}$ ), followed by continuous infusion at a rate of $6 \mathrm{~mL} / \mathrm{kg} / \mathrm{h}$. The dotted lines are the individual values, and the red solid lines are the locally weighted scatterplot smoothing (LOWESS) curves. In total, 20,084 segments were calculated with time ranging from 0 to $180 \mathrm{~min}$, and the number of points in the smoothing window was 10 . The time point at which $1000 \mathrm{~mL}$ of Ringer's lactate solution was initiated was set as the reference time 0

Table 3 shows the population pharmacokinetic parameter estimates and results of the non-parametric bootstrap replicates of the final pharmacokinetic model of Ringer's lactate solution.

The control stream of the final pharmacokinetic model that included the cardiac index and body weight is presented in the Supplementary file. Goodness-of-fit plots of the final pharmacokinetic model for Ringer's lactate solution are displayed in Fig. 3. Predictive checks of the final pharmacokinetic model are presented in Fig. 1b. In total, $10.4 \%$ of the data were distributed outside of the $90 \%$ prediction intervals of the predictive check, which indicates that the final model adequately described the time courses of plasma dilution. The simulated volume expansions of the central fluid space and peripheral fluid space in a hypothetical patient administered $10 \mathrm{~mL} / \mathrm{kg}$ of Ringer's lactate solution over $15 \mathrm{~min}$ followed by a rate of $8 \mathrm{~mL} / \mathrm{kg} / \mathrm{h}$ for $165 \mathrm{~min}$ are presented in Fig. 4. The estimated blood loss during operation was $134 \pm 92.8 \mathrm{~mL}$. Moreover, the urine output during the entire sampling period was $422.0 \pm 249.9 \mathrm{~mL}$.

\section{DISCUSSION}

As suggested by the final pharmacokinetic model, the elimination clearance $\left(k_{r}\right)$ increases with an increasing MAP. This finding is consistent with a previous study that showed a higher MAP was associated with a smaller volume expansion in the central and peripheral compartments [8]. Furthermore, as body weight increased, the central volume of the distribution at baseline increased, which is in line with the pharmacokinetic properties of other drugs [7, 18]. As body weight increases, the volume expansion due to fluid administration also increases. In a previous volume kinetic study, the central volume of distribution was shown to expand as the infused fluid increased with body weight [8].

Several studies have reported how to administer fluid in patients undergoing major abdominal surgeries, including open gastrectomy. Small trials have shown that a restrictive fluid regimen reduced the complication rate compared with a liberal fluid management regimen during elective surgery $[3,4]$. The restriction of fluid to achieve a zero balance is also an important element of the enhanced recovery after surgery (ERAS) protocol [19]; however, a recent study that compared liberal fluid therapy with restrictive fluid therapy in approximately 3000 patients undergoing abdominal surgery reported that restrictive therapy was not superior to liberal therapy, and it also increased the incidence of AKI [20]. Therefore, it is suggested that an adequate volume of fluid required for organ perfusion should be 
administered. The liberal fluid therapy performed in this study was as follows: a bolus of balanced salt crystalloid solution was administered at a dose of $10 \mathrm{~mL} / \mathrm{kg}$ of body weight during the induction of anesthesia, followed by a dose of $8 \mathrm{~mL} / \mathrm{kg} / \mathrm{h}$ until the end of surgery. Based on this regimen, the effect of volume expansion was simulated by considering the body weight and

Table 3. Population pharmacokinetic parameter estimates, interindividual variability, and median parameter values $(2.5 \%-97.5 \%)$ of the non-parametric bootstrap replicates of the final pharmacokinetic model for Ringer's lactate solution

\begin{tabular}{|c|c|c|c|}
\hline Parameters & $\begin{array}{l}\text { Estimates } \\
\text { (RSE, \%) }\end{array}$ & CV (\%) & Median (2.5\%-97.5\%) \\
\hline $\begin{array}{l}k_{r}(\mathrm{~mL} / \mathrm{min})=\theta_{1} \\
+(\mathrm{MAP} / 70)^{\theta 2}\end{array}$ & & \multicolumn{2}{|l|}{70.6} \\
\hline$\theta_{1}$ & $124(18.1)$ & & $116(90-149)$ \\
\hline$\theta_{2}$ & $14.2(9.6)$ & & $14.2(12.4-16.0)$ \\
\hline $\begin{array}{l}V_{c 0}(\mathrm{~mL})=\theta_{3} \\
+\theta_{4} \times(\mathrm{WT} / 63)\end{array}$ & & \multicolumn{2}{|l|}{23.0} \\
\hline$\theta_{3}$ & $0.95(4.2)$ & & $0.97(0.004-1.07)$ \\
\hline$\theta_{4}$ & $3440(12.8)$ & & $3280(2120-3810)$ \\
\hline$V_{t 0}(\mathrm{~mL})$ & $2730(32.1)$ & 38.0 & $3010(2050-4320)$ \\
\hline$k_{t}(\mathrm{~mL} / \mathrm{min})$ & $181(33.5)$ & - & $200(136-460)$ \\
\hline$\sigma$ & $0.00138(12.5)$ & - & $\begin{array}{l}0.0013 \\
(0.0011-0.0015)\end{array}$ \\
\hline \multicolumn{4}{|c|}{$\begin{array}{l}\text { A log-normal distribution of inter-individual random variability was } \\
\text { assumed. Residual random variability was modeled using an additive error } \\
\text { model. Non-parametric bootstrap analysis was repeated } 2000 \text { times. RSE } \\
\text { relative standard error }=\mathrm{SE} / \text { estimate } \times 100(\%), M A P \text { mean arterial pressure, } \\
\text { WT actual body weight, } k_{r} \text { elimination clearance }(\mathrm{mL} / \mathrm{min}), V_{c 0} \text { central } \\
\text { volume of distribution at baseline (mL), } V_{t 0} \text { peripheral volume of } \\
\text { distribution at baseline }(\mathrm{mL}), k_{t} \text { distributional clearance between central } \\
\text { and peripheral compartment }(\mathrm{mL} / \mathrm{min})\end{array}$} \\
\hline
\end{tabular}
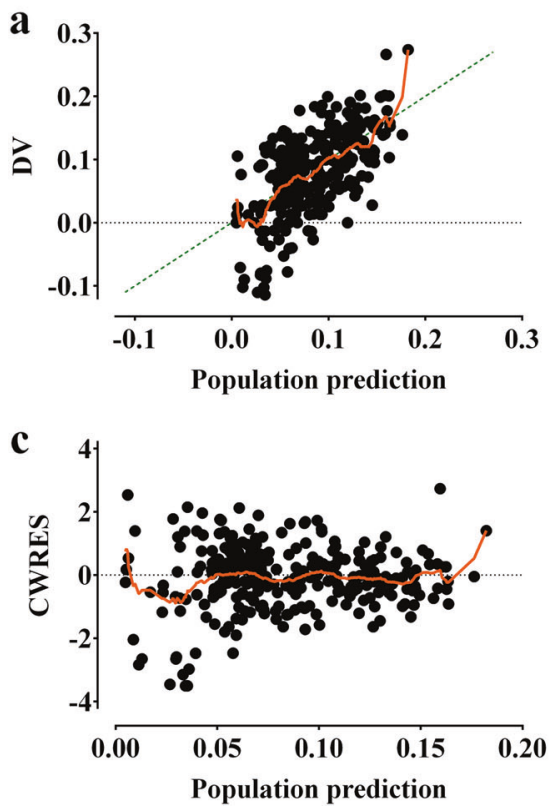

mean arterial pressure (Fig. 4). Assuming the same mean blood pressure, a patient who weighed $80 \mathrm{~kg}$ received twice as much fluid as a patient who weighed $40 \mathrm{~kg}$; however, the maximum volume expansion was $\sim 3$ times and the volume expansion at equilibrium was $\sim 4$ times greater in the central fluid space. These findings indicate that the volume expansion effect is not directly proportional to the infusion amount even when using a weightbased fluid infusion regimen. Even if the same amount of fluid is administered due to the same weight, the clearance rate of a patient with a high MAP would be higher, and consequently, the volume expansion effect is lower in both the central and peripheral fluid spaces. This suggests that not only the body weight but also the mean blood pressure should be taken into consideration when administering Ringer's lactate solution in clinical practice. The simulation results showed that when Ringer's lactate solution was administered at a dose of $10 \mathrm{mg} / \mathrm{kg}$ for a short period of time, approximately $50-65 \%$ of the infused fluids remained in the blood at the end of the administration, which is in line with the results from a previous study [5]. In addition, when Ringer's lactate solution was administered for a long time (e.g., $3 \mathrm{~h}$ ) at a rate of $8 \mathrm{~mL} / \mathrm{kg} / \mathrm{h}$ with the mean arterial pressure kept constant, the amount accumulated in the tissue was maintained at a constant level without increases over time. This simulation result is meaningful in that it enables a mathematical explanation of how Ringer's lactate solution is distributed to blood and tissues when the fluid is administered based on the liberal fluid regimen in patients undergoing open gastrectomy.

The estimated elimination clearance $\left(k_{r}\right)$ in this study was higher than the values reported in the existing literature [5]. This discrepancy is likely the result of the difference in the method of obtaining $k_{r}$, of which there are two methods-estimating $k_{r}$ from the structural model and calculating $k_{r}$ from the area under the time-dilution curve for the central compartment divided by the measured total urinary output [21]. In the studies conducted by Hahn RG, the latter method was used to obtain $k_{r}$. Among the volume kinetic parameters, $k_{r}$ was calculated using urine output data, and the remaining parameters $\left(V_{c 0}, V_{t 0}, k_{t}\right)$ were estimated from structural models using time-dilution data. It is not clear
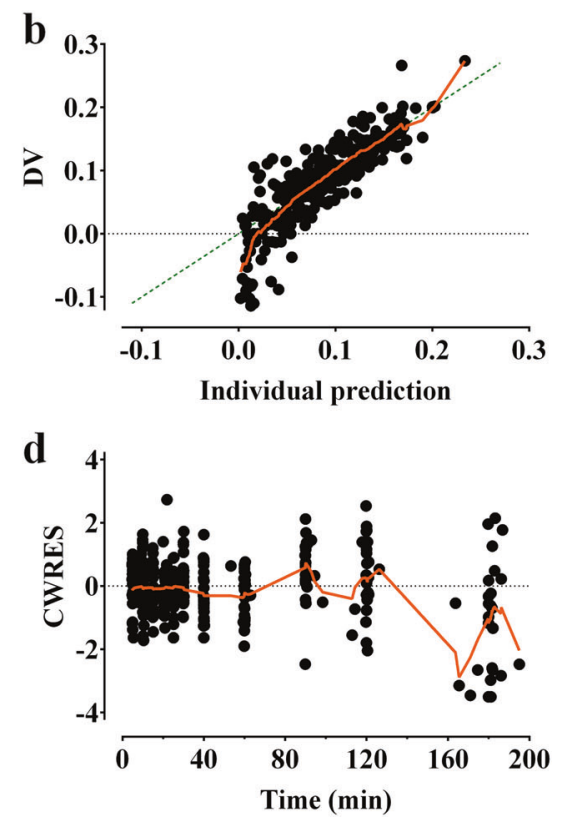

Fig. 3 Goodness-of-fit plots of the final pharmacokinetic model for Ringer's lactate solution. a Population prediction vs. plasma dilution, b individual prediction vs. plasma dilution, c conditional weighted residuals (CWRES) vs. population prediction, and d CWRES over time. The green dashed line is the line of identity. Locally weighted scatterplot smoothing (LOWESS) is shown as an orange solid line. In total, 1184 segments were calculated with prediction or time, and the number of points in the smoothing window was 10 (Prism 7 for Windows version 7.01; GraphPad Software Inc., San Diego, CA, USA) 

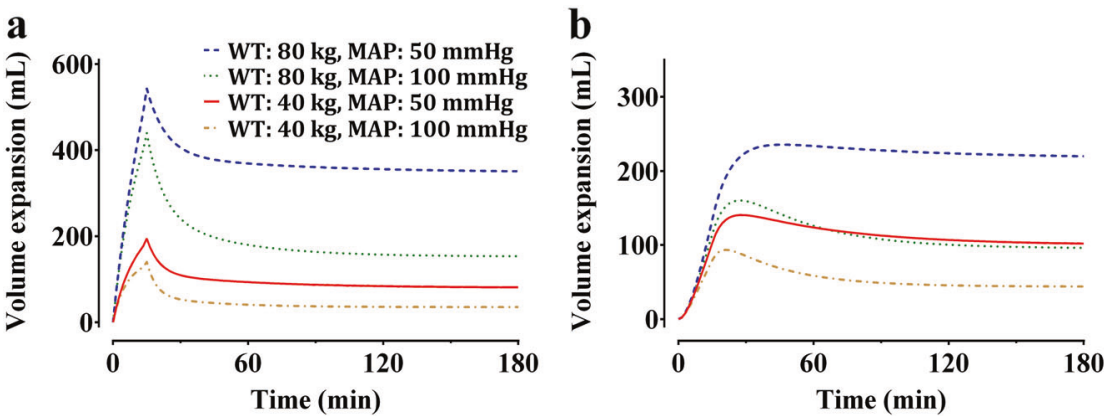

Fig. 4 Simulated volume expansions of the central fluid space (a) and peripheral fluid space (b) in a hypothetical patient receiving $10 \mathrm{~mL} / \mathrm{kg}$ of Ringer's lactate solution over $15 \mathrm{~min}$ followed by a rate of $8 \mathrm{~mL} / \mathrm{kg} / \mathrm{h}$ for $165 \mathrm{~min}$. Based on the weight $(40 \mathrm{vs}$. $80 \mathrm{~kg})$ and mean arterial pressure $(50$ vs. $100 \mathrm{mmHg})$, four different cases were simulated. WT weight, MAP mean arterial pressure

which method is more appropriate. However, we estimated $k_{r}$ in this study because we deemed that it was better to obtain all volume kinetic parameters from the same data rather than using different data to obtain different parameters. Since Hahn RG did not estimate $k_{r}$ from the structural model, the approximate value of $k_{r}$ estimated from time-dilution data was unknown. The median $(25-75 \%)$ value of $k_{r}$ calculated from the urine output data obtained in this study was $74.2(38.6-118.7) \mathrm{mL} / \mathrm{min}$. In addition, the type of operation, anesthetic method, patient's physical characteristics, and volume status at the time of fluid administration may be the cause of the higher $k_{r}$ value than the values obtained in previous studies [5, 21].

Recently, studies related to the volume kinetics of fluids have attempted to estimate $k_{12}$ (distributional clearance from the central compartment to peripheral compartment) and $k_{21}$ (distributional clearance from the peripheral compartment to central compartment) individually rather than estimating $k_{\mathrm{d}}$ (distributional clearance) $[8,9,22]$. However, in this study, $k_{\mathrm{d}}$ was estimated using a conventional method for three reasons. First, the Akaike Information Criteria of the model including $k_{12}$ and $k_{21}$ was not better than the model containing $k_{\mathrm{d}}$ (model including $k_{12}$ and $k_{21}$ : -1531.1 , model including $k_{d}$ : -1533.7$)$. Second, it may be more reasonable to estimate $k_{\mathrm{d}}$ physiologically. In the case of drugs, the active ingredient of a drug can be considered to move between the central compartment and the peripheral compartment, and the concentration is measured to characterize the pharmacokinetics of the drug. However, in the volume kinetics of fluids, water moves between compartments and the hemoglobin concentration is measured. Moreover, hemoglobin is present only in the blood vessels and not in the tissues. It may not be appropriate to estimate $k_{21}$ by measuring the hemoglobin concentration. Because water moves between compartments, $k_{12}$ and $k_{21}$ are likely similar. Third, in a previous study, when $k_{12}$ and $k_{21}$ were independentely estimated, $k_{21}$ showed a negative value [9] The authors interpreted this as an accumulation of fluid in the interstitium; however, it appears to be a somewhat arbitrary interpretation in terms of the pharmacokinetic model.

As shown in Fig. 1a, negative values of the plasma dilution were observed in approximately $44 \%$ of patients, which suggests that the hemoglobin was higher than the baseline value during the sampling period. This can be interpreted as hemoconcentration, as Ringer's lactate solution was administered in a manner similar to restrictive fluid management during surgery. Because the fluid was administered at the minimum amount required and the urine output was relatively high, hemoconcentration may have occurred in some patients. However, because the structural model of volume kinetics evaluates the amount of volume expansion that has been induced by fluid administration compared to the baseline, it was structurally unpredictable to reduce from the initial volume (refer to Supplementary file). Thus, the volume kinetic model cannot account for hemoconcentration, which was also observed in a previous study [22]. This may be the main cause of the poor goodness-of-fit plot when the plasma dilution was negative (Fig. 3).

There were several limitations to this study. First, the amount of intraoperative bleeding was not included in the volume kinetic model. It is reasonable to use hemoglobin as an endogenous tracer when characterizing the kinetics of fluids under the basic premise that hemoglobin is not lost [5]. However, there is no method for reflecting the amount of bleeding in the structural model of volume kinetics. The amount of bleeding in this study was low compared to the total blood volume; thus, it is not clear whether this factor had a large effect on the calculation of the plasma dilution. In previous studies, plasma expansion was well described using volume kinetic models in surgical patients undergoing Cesarean section or colon surgery [23, 24]. Second, volume kinetic parameters may vary depending on whether a patient is anesthetized or conscious. The elimination clearance $\left(k_{r}\right)$ was lower in anesthetized patients than in awake volunteers [5]. Vasodilation induced by anesthetic agents was thought to be responsible for most of this decrease [5]. In this study, the last blood sample (180 min after the time of initiating the administration of $1000 \mathrm{~mL}$ of Ringer's lactate solution) of all patients was collected in the postanesthesia care unit. Although the mixed states of the anesthetic state and arousal state may affect the volume kinetic parameters, this did unlikely have a substantial influence because only one point per patient was collected in the consciousness state.

In conclusion, the two-volume model well described the change in hemoglobin-derived plasma dilution induced by the administration of Ringer's lactate solution in surgical patients undergoing open gastrectomy. A higher cardiac index was associated with a greater elimination clearance and, consequently, less water accumulation in the interstitium. As the body weight increased, the volume expansion in the blood vessels increased. These results can be used as a reference for the administration of Ringer's lactate solution based on the patient's physical characteristics.

\section{ACKNOWLEDGEMENTS}

This study was supported by a Student Research Grant (17) from the University of Ulsan College of Medicine, Seoul, Korea. We thank Dr. Joon Seo Lim from the Scientific Publications Team at Asan Medical Center for his editorial assistance in preparing this manuscript.

\section{AUTHOR CONTRIBUTIONS}

J.-H.L., Y.-J.C., B.-M.C., and S.-H.L. designed the study; J.-H.R., Y.-H.L., S.T.Oh., and K.-T.C. collected the data; J.-H.L., Y.-J.C., B.-M.C., S.-H.L., and G.-J.N. performed the data 
analysis and interpretation. All authors contributed to the writing of the manuscript, provided critical revisions, and approved the final version.

\section{ADDITIONAL INFORMATION}

The online version of this article (https://doi.org/10.1038/s41401-018-0179-3) contains supplementary material, which is available to authorized users.

Competing interests: The authors declare no competing interests.

\section{REFERENCES}

1. Aga Z, Machina M, McCluskey SA. Greater intravenous fluid volumes are associated with prolonged recovery after colorectal surgery: a retrospective cohort study. Br J Anaesth. 2016;116:804-10.

2. Li Y, He R, Ying X, Hahn RG. Ringer's lactate, but not hydroxyethyl starch, prolongs the food intolerance time after major abdominal surgery; an open-labelled clinical trial. BMC Anesthesiol. 2015;15:72.

3. Schol PB, Terink IM, Lance MD, Scheepers HC. Liberal or restrictive fluid management during elective surgery: a systematic review and meta-analysis. J Clin Anesth. 2016;35:26-39.

4. Weinberg L, lanno D, Churilov L, Chao I, Scurrah N, Rachbuch C, et al. Restrictive intraoperative fluid optimisation algorithm improves outcomes in patients undergoing pancreaticoduodenectomy: a prospective multicentre randomized controlled trial. PLOS ONE. 2017;12:e0183313.

5. Hahn RG. Volume kinetics for infusion fluids. Anesthesiology. 2010;113:470-81.

6. Stahle L, Nilsson A, Hahn RG. Modelling the volume of expandable body fluid spaces during i.v. fluid therapy. Br J Anaesth. 1997;78:138-43.

7. Choi BM, Lee YH, An SM, Lee SH, Lee EK, Noh GJ. Population pharmacokinetics and analgesic potency of oxycodone. Br J Clin Pharmacol. 2017;83:314-25.

8. Hahn RG. Arterial pressure and the rate of elimination of crystalloid fluid. Anesth Analg. 2017;124:1824-33.

9. Li Y, Xiaozhu Z, Guomei R, Qiannan D, Hahn RG. Effects of vasoactive drugs on crystalloid fluid kinetics in septic sheep. PLoS ONE. 2017;12:e0172361.

10. Kim YK, Chin JH, Kang SJ, Jun IG, Song JG, Jeong SM, et al. Association between central venous pressure and blood loss during hepatic resection in 984 living donors. Acta Anaesthesiol Scand. 2009;53:601-6.
11. Beal S, Sheiner L. NONMEM user's guides introduction to NONMEM 7 Version 7.1.2. San Francisco: NONMEM Project Group, University of California; 2009. p. 9-10.

12. Svensen C, Hahn RG. Volume kinetics of Ringer solution, dextran 70, and hypertonic saline in male volunteers. Anesthesiology. 1997;87:204-12.

13. Mosteller RD. Simplified calculation of body-surface area. N Engl J Med. 1987; 317:1098.

14. Janmahasatian S, Duffull SB, Ash S, Ward LC, Byrne NM, Green B. Quantification of lean bodyweight. Clin Pharmacokinet. 2005;44:1051-65.

15. Robinson JD, Lupkiewicz SM, Palenik L, Lopez LM, Ariet M. Determination of ideal body weight for drug dosage calculations. Am J Hosp Pharm. 1983;40: 1016-9.

16. Choi BM, Lee HG, Byon HJ, Lee SH, Lee EK, Kim HS, et al. Population pharmacokinetic and pharmacodynamic model of propofol externally validated in children. J Pharmacokinet Pharmacodyn. 2015;42:163-77.

17. Wahlby U, Jonsson EN, Karlsson MO. Assessment of actual significance levels for covariate effects in NONMEM. J Pharmacokinet Pharmacodyn. 2001;28: 231-52.

18. Marsh B, White M, Morton N, Kenny GN. Pharmacokinetic model driven infusion of propofol in children. Br J Anaesth. 1991;67:41-8.

19. Fearon KC, Ljungqvist $\mathrm{O}$, Von Meyenfeldt $\mathrm{M}$, Revhaug A, Dejong $\mathrm{CH}$, Lassen $\mathrm{K}$, et al. Enhanced recovery after surgery: a consensus review of clinical care for patients undergoing colonic resection. Clin Nutr. 2005;24:466-77.

20. Myles PS, Bellomo R, Corcoran T, Forbes A, Peyton P, Story D, et al. Restrictive versus liberal fluid therapy for major abdominal surgery. N Engl J Med. 2018; 378:2263-74.

21. Olsson J, Svensen CH, Hahn RG. The volume kinetics of acetated Ringer's solution during laparoscopic cholecystectomy. Anesth Analg. 2004;99:1854-60. Table of contents.

22. Hahn RG, Drobin D, Zdolsek J. Distribution of crystalloid fluid changes with the rate of infusion: a population-based study. Acta Anaesthesiol Scand. 2016;60: 569-78.

23. Hahn RG, Resby M. Volume kinetics of Ringer's solution and dextran 3\% during induction of spinal anaesthesia for caesarean section. Can J Anaesth. 1998;45: 443-51.

24. Ewaldsson CA, Hahn RG. Volume kinetics of Ringer's solution during induction of spinal and general anaesthesia. Br J Anaesth. 2001;87:406-14. 